\title{
Femmes indiennes entre nationalisme et féminisme, des années 1880 à 1947
}

Indian Women between nationalism and feminism (1880s-1947)

\section{Nupur Chaudhuri}

Traducteur : Anne Hugon

\section{(2) OpenEdition Journals}

\section{Édition électronique}

URL : https://journals.openedition.org/clio/10017

DOI : 10.4000/clio.10017

ISSN : $1777-5299$

\section{Éditeur}

Belin

Édition imprimée

Date de publication : 1 mai 2011

Pagination : 85-106

ISBN : 978-2-8107-0157-5

ISSN : 1252-7017

\section{Référence électronique}

Nupur Chaudhuri, «Femmes indiennes entre nationalisme et féminisme, des années 1880 à 1947 », Clio. Femmes, Genre, Histoire [En ligne], 33 | 2011, mis en ligne le 01 mai 2013, consulté le 27 avril 2022. URL : http://journals.openedition.org/clio/10017 ; DOI : https://doi.org/10.4000/clio.10017 


\title{
Femmes indiennes entre nationalisme et féminisme, des années 1880 à $1947^{1}$
}

\author{
Nupur CHAUDHURI
}

En 1947, l'indépendance de l'Inde met un terme aux luttes pour l'émancipation complète vis-à-vis de la tutelle britannique. Du côté indien, des femmes comme des hommes ont participé à cette résistance depuis la fin du XIX ${ }^{e}$ siècle. Cet article, centré sur l'engagement des femmes dans le mouvement nationaliste, examine leur rôle à la fois dans les luttes violentes et non-violentes. À partir de l'étude des mémoires, autobiographies et journaux rédigés en bengali, en anglais et en hindi, on s'intéresse en particulier à l'articulation entre revendications religieuses, féministes et nationalistes de la part de femmes qui furent actives dans la première moitié du XXe siècle².

Depuis les années 1990, aussi bien en Inde qu'en Occident, on assiste à une abondance de recherches féministes qui placent l'histoire des Indiennes au centre de l'histoire de l'Asie du Sud comme de l'histoire des femmes ${ }^{3}$. Ces travaux montrent que, contrairement à une idée reçue aussi bien en Occident qu'en Asie du Sud, les femmes indiennes, loin d'être des sujets passifs dans le processus d'indépendance, s'y sont impliquées; et que leur investissement a eu un impact sur les caractéristiques du mouvement nationaliste. Cependant, la plupart des études entreprises en Occident se sont appuyées sur des sources en anglais, plus faciles d'accès, mais

1 Les responsables du numéro tiennent à remercier Rebecca Rogers pour son aide précieuse lors de l'édition de ce texte.

2 Les traductions du bengali sont de Nupur Chaudhuri.

3 Parmi de nombreux travaux, voir en particulier : Basu \& Ray 1990 ; Borthwick 1984 ; Ray 1995 ; Sarkar 1999, 2001 ; Thapar-Björkert 2006 ; Burton 1998 ; Forbes 1998 ; Ramusack 1981 ; Sinha 1996. 
produites par des femmes éduquées à l'occidentale et qui reflètent donc le contexte impérial dans lequel elles ont émergé 4 . Une poignée seulement de travaux en anglais se sont penchés sur des sources en langues indiennes, bien que la plupart des Indiennes n'aient pas utilisé l'anglais dans leurs écrits ${ }^{5}$. Par conséquent, les recherches se sont surtout concentrées sur la façon dont les femmes britanniques élaboraient et développaient leur propre vision du féminisme et du nationalisme, vision influencée par l'idée de l'infériorité des femmes indiennes ${ }^{6}$.

L'analyse du discours des nationalistes indiennes permet une autre lecture. Elle montre que les femmes du mouvement nationaliste usent de métaphores familiales et se représentent en mères de la nation dans leur combat contre les Britanniques, puisant dans la même rhétorique que leurs consœurs occidentales ${ }^{7}$. Le discours nationaliste indien décrit l'Inde comme la mère-patrie (Bharatmata) et les femmes comme des mères dont le rôle est double: elles engendrent les enfants mais se doivent aussi d'être gardiennes de la foi et des rituels religieux. La mère-patrie est considérée comme un corps de femme violé par des hommes étrangers (les Britanniques) par le biais de la colonisation. Dès lors, pour restaurer l'honneur de l'Inde, il faut que des Indiens se sacrifient. Cette conception de la maternité symbolique a pris une grande importance dans la définition du nationalisme

4 Burton 2003 ; Grewal 1996.

5 Krishnobhabini Das, par exemple, écrit en bengali : seuls ceux qui comprennent cette langue connaissent donc ses travaux et sa contribution au mouvement nationaliste. Voir Chaudhuri 2010. Ainsi que Angol 2006; Kosambi 2000 ; Sindhe 1994 ; Sarkar 1993, 1999, 2001 ; Thapar-Björkert 2006 ; Sreenivas 2008.

6 Burton 1994 ; Chaudhuri 2000. Le premier ouvrage qui se soit intéressé à l'interaction entre Européennes et femmes indigènes a été le livre édité par Chaudhuri \& Strobel 1992. Depuis lors, Burton a publié plusieurs ouvrages sur les liens entre femmes britanniques et indiennes; et Chaudhuri, plusieurs articles sur la vie des Britanniques en Inde. Par ailleurs, Levine d'une part (2004) et Woollacott d'autre part (2006) ont analysé l'interaction entre Européens et populations indigènes. On peut lire le point de vue de See Urvashi Butalia sur l'influence britannique en Inde après 1947 (Levine 2004). Woollacott n'utilise que des sources en anglais.

7 Voir Blom $2000: 8$. 
indien et devient même un symbole de l'identité indienne. Dans l'ensemble, hommes et femmes des classes moyennes et supérieures, loin d'associer la maternité à une position subordonnée, y voient un moyen de contribuer au mouvement d'émancipation ${ }^{8}$. Non seulement les femmes acceptent de passer pour des icônes du nationalisme mais elles s'engagent activement dans le mouvement de libération. S'identifiant aux combattants de la liberté d'autres pays opprimés, Sarojini Naidu, devenue présidente du Parti du Congrès en 1928, écrit en 1931 :

Je suis une guerrière indienne qui se bat pour la liberté de son pays. Vous tous, vous luttez pour protéger votre mère-patrie. En d'autres termes, notre cause est la même et nous appartenons donc tous à la même famille?

\section{Les traces d'une résistance précoce des femmes}

Des femmes s'engagent dès les débuts de la résistance contre les Britanniques. La figure la plus emblématique de ce combat précoce est peut-être celle de Rani Lakshmi Bai, reine de l'État princier du Jhansi, situé en Inde centrale. Née en 1827, elle épouse le souverain du Jhansi en 1842. Ayant perdu leur unique enfant en bas âge, elle et son époux adoptent un fils supposé hériter du trône. Le roi meurt alors que cet héritier n'a que cinq ans, laissant la régence à la reine. Lorsque Lakshmi Bai en informe les Britanniques, le Gouverneur général décide de révoquer l'adoption. Ayant fait appel de cette décision à plusieurs reprises, Rani Lakshmi Bai est systématiquement déboutée. En 1857, durant la mutinerie provoquée par la nouvelle selon laquelle les balles de mitrailleuses sont enduites de graisse de porc ou de bœuf, les troupes indiennes du Jhansi se joignent à la rébellion contre les Britanniques. Au même moment, la reine conclut une alliance avec des souverains rebelles et recrute des soldats parmi les révoltés, afin de résister à l'attaque d'un royaume voisin qui a des vues sur son trône. Lorsque les Britanniques attaquent le Jhansi, elle quitte le fort avec son fils et rejoint des chefs de la rébellion avec ses soldats. Non loin du fort de Gwalior (Inde centrale), en habit

8 Thapar-Björkert $2006: 83-87$.

9 Vartman, 18 octobre $1931: 3$. Également cité par Thapar-Björkert $2006: 87$. 
d'homme, elle prend la tête de ses troupes dans leur assaut contre les Britanniques. Elle meurt sur le champ de bataille le 17 juin 1857, gagne le statut de martyr et devient immédiatement, dans les milieux de l'art de la littérature populaire, une figure de légende. Durant les trois premières décennies du $\mathrm{XX}^{\mathrm{e}}$ siècle, plusieurs leaders nationalistes, qui ont opté pour la lutte armée révolutionnaire comme voie vers l'indépendance, la considèrent comme une héroïne nationale, avant qu'elle ne devienne une figure féministe de premier plan après $1947^{10}$.

À partir de 1880, le mécontentement grandissant à l'égard des Britanniques se fait plus évident à la fois dans les écrits et dans les faits, notamment parmi les femmes instruites des classes moyennes et supérieures bengalies, issues de familles engagées dans la lutte contre la tutelle britannique. Parmi elles, on retiendra surtout Krishnobhabini Das et Sarala Devi Chaudhurani. L'ouvrage de la première, publié en 1885, Englande Bangamobila (Une Bengalie en Angleterre $)^{11}$, se présente comme un récit de voyage mais son objectif est de créer une identité nationaliste parmi les Bengalies de la classe moyenne et de développer chez elles un esprit féministe par la promotion de l'instruction. Les Britanniques ne s'y trompent pas, et interdisent ce livre qui promeut à la fois l'idéologie nationaliste et féministe dans ce milieu ${ }^{12}$.

Parallèlement, la résistance politique commence à émerger vers 1883-1884, à l'occasion de la controverse sur le décret Ilbert, qui permettait à des juges indiens de juger des Européens, au grand dam de nombre de ces derniers. Ce projet suscite des sentiments antibritanniques parmi les Indiens, en particulier parmi les Bengalis mobilisés contre les opposants à ce décret. Ainsi, comme les hommes, des femmes des classes moyennes et supérieures se sont investies dans les mouvements anti-britanniques avant même la création du Parti du Congrès, qui allait rejoindre peu après le mouvement à l'échelle nationale. Lorsque Surendranath Banerjea, l'un des chefs du jeune Congrès, est condamné à la prison pour outrage à

\footnotetext{
10 Chapman-Lebra 1986 : 104-115, 142-143, 151-152.

11 Das 1885.

12 Chaudhuri $2010: 145$
} 
la cour, son emprisonnement et la révolte engendrée par les débats sur le décret Ilbert encouragent la mobilisation politique des femmes bengalies, qui descendent dans la rue en 1883 pour démontrer leur soutien à Banerjea. Elles organisent des réunions en faveur du décret et leurs mémoires révèlent bien le rôle de catalyseur joué par cet événement. Ainsi, les enseignantes de l'école de filles Bethune manifestent pour soutenir le décret; l'une d'elles organise de nombreux meetings destinés aux élèves, lesquelles se mettent à porter des badges de soutien ${ }^{13}$. Dans la foulée de cette mobilisation, la création du Congrès National Indien en 1885 marque le début d'une activité politique au niveau national. En 1889, on compte six femmes parmi les délégués qui assistent à la réunion annuelle du parti à Bombay ${ }^{14}$.

Cette participation des femmes bengalies au mouvement nationaliste provient en partie de réformes promues par les hommes de l'élite hindoue (les bhadraloka bengalis). Durant les années 1880, tandis que s'organise le Parti du Congrès, ils œuvrent pour réformer le statut social des femmes et encourager leur instruction. Leur but est de permettre aux épouses de mieux soutenir socialement leur mari et d'être mieux à même d'élever des fils instruits. Les bhadraloka souhaitent que leurs femmes adoptent le comportement victorien sans abandonner les valeurs traditionnelles des femmes indiennes ${ }^{15}$. Comme dans bien d'autres luttes nationalistes, les hommes exigent des femmes qu'elles sachent transmettre les traditions et la religion ${ }^{16}$. Dans ce but, les leaders traditionalistes bengalis s'appuient sur les luttes socio-économiques pour promouvoir le concept de suprématie de la mère-patrie, insistant sur le pouvoir incarné par les déessesmères (kali et shakti). À l'instar de Tagore, des intellectuels magnifient cette image de déesse-mère ou shakti, concept pré-aryen au Bengale, afin de conférer une dimension religieuse à la mobilisation nationaliste, encourageant ainsi les femmes à y participer puisqu'elles relèvent du shakti. L'une des conséquences de cette

\footnotetext{
13 Chaudhurani $1975: 28,216$.

14 Bahmabodhini Patrika, mai 1890, 4/4, p. 304.

15 Ray $1995: 179$.

16 Voir Anthias \& Yuval-Davis $1989: 7$.
} 
conception hindoue du nationalisme est qu'elle décourage certains musulmans de s'y associer ${ }^{17}$.

\section{La résistance contre la tutelle britannique au Bengale, 1905-1920}

La réforme du statut des femmes au Bengale explique en partie leur participation accrue à la lutte contre les Britanniques au début du $\mathrm{XX}^{\mathrm{e}}$ siècle. Le projet de partition du Bengale par Lord Curzon suscite une très large opposition des Hindous bengalis en 1905. Parmi eux, les leaders du Parti du Congrès en appellent à la population (notamment aux femmes) pour boycotter les produits britanniques et acheter des produits indiens. Les journaux bengalis se mettent à publier des réclames pour du savon, de l'huile capillaire et autres biens de consommation domestique fabriqués en Inde. Le 16 octobre 1905, jour de la partition, des femmes de toute la région se mobilisent pour protester : ainsi, en signe de soutien au mouvement nationaliste, nombre d'enseignantes chrétiennes se rendent au travail pieds nus. Mais, dans l'ensemble, les musulmans soutiennent ce projet, qui leur permet de devenir majoritaires au Bengale oriental.

Au même moment, coexistant avec la résistance non-violente, des sociétés révolutionnaires animées par de jeunes hommes voient le jour au Bengale. Entre 1905 et 1910, les femmes demeurent rares dans ces associations. Mais de nombreuses femmes des classes moyennes soutiennent les révolutionnaires, en les cachant pour les soustraire à la police, ou encore en agissant comme messagères ou porteuses d'armes. En 1905, des femmes hindoues du Bengale, citadines ou rurales, rejoignent le mouvement. Le 15 novembre 1908, le Daily Telegraph (à Londres) publie un article dans lequel on peut lire que les Bengalies « étaient les adversaires les plus obstinées et les plus dangereuses des Britanniques». À cette époque, les femmes participent aussi en diffusant des chansons patriotiques et en jouant dans des pièces de théâtre nationalistes. La riposte du Raj, conformément aux souhaits des Britanniques, est d'interdire les représentations théâtrales et les chants patriotiques ${ }^{18}$.

\footnotetext{
17 Basu \& Ray $1990: 183$.

18 Ray $1995: 189$.
} 
Sarala Devi Chaudhurani (1872-1945) est la première femme à rejoindre le mouvement révolutionnaire, pour en devenir rapidement l'une des leaders. Par sa mère, sœur aînée du Prix Nobel de littérature Rabindranath Tagore, elle appartient à la famille Tagore, dont l'apport à la vie intellectuelle bengalie aux XIX ${ }^{\mathrm{e}}$ et $\mathrm{XX}^{\mathrm{e}}$ siècles fut immense. Chaudhurani développe des contacts avec des révolutionnaires bengalis (biplabis) en créant gymnasiums et clubs de culture physique et en animant des groupes de jeunes. Après son mariage, installée au Punjab, elle diffuse les idées nationalistes, en éditant un périodique bengali intitulé Bharati, fondé et financé par la famille Tagore. Elle maintient également des liens avec une société révolutionnaire fondée en $1900^{19}$.

La mobilisation des Bengalies dans le mouvement nationaliste est également influencée par leurs contacts avec des féministes étrangères, comme Margaret Noble (1867-1911), irlandaise protestante et militante politique et sociale. Arrivée à Kolkata (Calcutta) en 1897, elle entre dans une communauté monastique hindoue et y prend le nom de Sœur Nivedita. En 1898, elle ouvre une école pour filles où est prise en compte la culture hindoue et dans laquelle elle enseigne en bengali. Son implication dans le mouvement nationaliste indien la pousse à quitter le monastère en 1902, lorsqu'elle devient membre de l'Anushilan Samiti, l'une des sociétés révolutionnaires du Bengale, d'où elle inspire plus d'un jeune révolutionnaire. Repartie en Angleterre en 1907 pour revenir en 1909, elle soutient clandestinement la cause nationaliste au Bengale jusqu'à sa mort en $1911^{20}$. Au début du XX ${ }^{\text {e }}$ siècle, des féministes comme Annie Besant ou l'Irlandaise Margaret Cousins encouragent les Bengalies à s'engager pour les droits des femmes². En 1914, la

19 Chaudhurani 1975 ; Ray 2002.

20 Ramusack 1992 : 125 ; Basu 1985 : 25-26 ; Pravarajika 1967.

21 Annie Besant (1847-1933) est une féministe britannique, adepte de la théosophie. Margaret Cousins (1878-1954) a suivi son mari, parti à Madras comme journaliste. En 1916, elle fonde la Société pour l'Amélioration de la condition du sexe faible, qui devient un modèle pour diverses associations de femmes indiennes. Avec Annie Besant, elle crée en 1917 une autre association destinée à encourager la participation des femmes à la vie publique. En 1926, elle contribue à la naissance du Congrès des Femmes Indiennes (All India Women's 
première est déjà fortement impliquée dans le mouvement nationaliste et a même fait de la prison pour avoir créé sur place une Ligue pour l'Autonomie (Home Rule League) sur le modèle irlandais. À sa sortie de prison, elle est élue à la présidence du Congrès National Indien. Son implication politique pousse de nombreuses Indiennes, notamment bengalies, à s'investir à leur tour dans le mouvement nationaliste. D'autant plus que durant son mandat, le Congrès tient ses réunions annuelles à Kolkata, entraînant un nombre croissant de femmes du Bengale engagées dans le mouvement swadeshi22.

La récession mondiale consécutive à la Première Guerre mondiale n'épargne pas l'Inde: dans les années 1920, le prix des produits agricoles baisse nettement, tandis qu'augmentent les loyers et les intérêts, affectant durement pauvres et riches tout à la fois. Cette période de dépression économique se traduit par la fermeture de nombreuses usines, entraînant une agitation politique croissante dans le centre et le Nord du pays. Des membres de toutes les classes sociales s'impliquent plus fortement dans la vie politique et rejoignent le Parti du Congrès - et parmi eux, bon nombre de femmes, qui se joignent au mouvement pour une multitude de raisons, qui dépassent les seules motivations économiques.

\section{Les femmes et la non-violence dans les années 1920 et 1930}

Tout d'abord, les femmes de l'élite du Bengale et du Maharashtra s'efforcent d'encourager celles des classes moyennes à se joindre à la mobilisation. En outre, les leaders du Congrès font tout pour que les femmes participent au mouvement de non-coopération dirigé contre la tutelle britannique. Ils savent que sans la mobilisation des masses, et notamment des femmes de toutes les classes, la politique de noncoopération sera un échec au lieu d'une force démontrant l'unité

Conference), destiné à améliorer l'instruction des femmes, et dont elle prend la tête en 1936 avant de se retirer de la vie publique en 1943. Voir Cousins \& Cousins 1950. À propos de l'influence irlandaise sur la résistance indienne et bengalie après la Première Guerre mondiale, voir Silvestri 2000.

22 Ray $1995: 190$. 
nationale. Gandhi lui-même pousse les hommes à laisser les femmes de leur famille prendre part à cet effort ${ }^{23}$.

Une spécialiste du mouvement nationaliste indien distingue trois catégories de femmes engagées dans la lutte nationaliste de ces années-là. D'abord, des femmes qui, sans appartenir à aucun parti politique, prennent part aux piquets de boycott ou satyagrahas. Ensuite, celles qui optent pour certains aspects du programme de Gandhi : vivre dans un ashram, participer à la campagne du khadi (le tissu de coton indien), boycotter les produits étrangers... Enfin, un petit groupe de femmes d'élite, activement engagées dans le mouvement nationaliste et régulièrement incarcérées ${ }^{24}$. Dans toute l'Inde, des femmes de diverses classes et castes rejoignent le mouvement de non-coopération, bien que dans certaines régions comme le Sud, leur participation soit restée limitée. En dépit d'un fort taux d'illettrisme dans certaines zones, des femmes adhèrent au Parti, tandis que d'autres prennent part aux mobilisations. Dans le nord du pays, la famille Nehru organise et entretient le mouvement de noncoopération, mais en l'absence de toute organisation politique féminine, seules des femmes de l'élite et des classes supérieures y participent: cela est plus facile pour elles, du fait de l'implication politique de leur famille et des encouragements des hommes de leur entourage.

Le 7 décembre 1921, Basanti Debi, l'épouse de C.R. Das, chef de l'aile gandhienne de la non-coopération au Bengale, est arrêtée avec d'autres, alors qu'elles essayaient de vendre du khaddar (cotonnade brute tissée à la main) pour protester contre l'importation de textile anglais. Bien que leur arrestation n'ait duré que quelques heures, l'une d'entre elles affirme: "Nous avions ainsi servi d'exemple pour les autres femmes: notre arrestation avait produit l'effet désiré ${ }^{25}$. Pendant l'incarcération de son mari, C.R. Das, devenu entre-temps président du Congrès de la province du Bengale, Basanti Debi prend la tête de l'organisation et en préside les réunions (1921-1922). En 1922, Hemaprobha Majumdar fonde le Mahila Karmi Sansad (Forum

25 Bangalar Katha (Histoire du Bengale), 16 décembre 1921.
} 
des Femmes Travailleuses), destiné à donner une formation professionnelle ainsi que politique. Alors qu'elle mène une manifestation de femmes, elle est blessée dans une échauffourée avec la police. L'année 1922 voit l'arrestation de nombreuses femmes qui manifestent dans les rues ou tiennent des piquets devant les boutiques vendant des produits britanniques.

Ces activités font partie intégrante du mouvement de noncoopération. Parmi les événements marquants, figure l'arrestation et la condamnation de Savitri Devi, soumise à une peine de trois mois de prison, qui fait d'elle la première femme engagée politiquement à être sévèrement condamnée. La presse indigène (comme l'Hindustan des 8 décembre 1921 et 9 février 1922) fait remarquer que les nombreuses incarcérations de femmes ne font qu'alimenter la lutte anticoloniale car la population est très sensible à l'humiliation qui leur est faite. Dans un rapport à ses supérieurs, le responsable de la Police de Calcutta prévient qu'à moins de prendre des mesures urgentes, le mouvement lancé par les femmes ne fera que croître; il craint « une collision entre les agitatrices et les forces de police $»^{26}$. Dans ces années-là, seule une poignée de femmes musulmanes prend part au mouvement de non-coopération; Daulatunnessa Khatun est l'une des exceptions à la règle, établissant des associations de femmes (samitis) dans des villages reculés du Bengale pour y relayer le mot d'ordre de Gandhi et le mouvement de Désobéissance Civile ${ }^{27}$.

C'est en 1928 que les Bengalies commencent à s'intéresser plus massivement à la lutte pour la liberté, lors de la réunion annuelle du Parti du Congrès. Subhas Bose, alors simple membre du parti dont il deviendra président en 1938 et 1939, décide que, comme les hommes, les femmes volontaires doivent porter l'uniforme et marcher au pas. Latika Ghosh (qui a fondé le Mahila Rashtriya Sangha en 1928, affilié au Congrès mais sans en faire partie) prend en charge le recrutement de femmes volontaires dans les établissements d'enseignement, notamment parmi les institutrices de Kolkata. Les villes de province

26 « Note sur les agitatrices », par R. Clarke, CIE, Commissaire de Police, Calcutta, 12 Janvier 1922, (Govt. of Bengal, Home Political File n²8, 1-8, 1922, West Bengal State Archives, Calcutta).

27 Amin $1995: 128$. 
suivent. L'organisation de Ghosh a pour double objectif l'indépendance du pays et l'égalité avec les hommes. En 1929, des Bengalies créent le Nari Satyagraha Samiti ainsi que d'autres associations, pour permettre aux femmes des classes moyennes urbaines de participer au boycott et aux marches associées au mouvement de non-coopération. Dans les années 1930, de nombreuses étudiantes rejoignent le mouvement nationaliste. Pour les y encourager, Sarojini Naidu, présidente du Congrès en 1928, écrit :

Jusqu'à présent nous avons été spectatrices : désormais, il faut agir. Vous savez toutes quel est votre devoir. Vous devez vous débarrasser du trône de Grande-Bretagne. Ne vous prenez pas pour des petites filles. Vous êtes les puissantes Durgas qui avancez masquées. Vous entonnerez les chants nationalistes partout où vous irez. Vous romprez nos chaînes. Vous libérerez votre pays. Qu'importe la terre: vous déplacerez les cieux. $^{28}$

Dans d'autres régions, des femmes s'engagent aussi dans le mouvement de non-coopération. À Bombay, elles transmettent des informations sur le mouvement au travers des Comités de District du Congrès ou encore des Bureaux de Volontaires du Congrès, dont elles sont membres. Comme au Bengale, elles créent leur propre organisation politique, la Rashtriya Stree Sabha. Sous la présidence de Sarojini Naidu et la vice-présidence de Goshiben Naoroji, une parsie, nombre de chrétiennes et de parsies rejoignent l'organisation.

Au Gujarat, l'État où est né Gandhi, les femmes participent également à la lutte : de nombreuses militantes sont instruites dans la langue vernaculaire et connues localement sous le terme de 《intelligentsia féminine $»^{29}$. En plus de leur investissement dans le boycott du sel, elles enjoignent les paysans à ne pas payer les frais de pâturage ou encore à couper de l'herbe et du bois dans les zones réservées. Au Bengale et au Maharashtra, on observe des initiatives similaires. Quand les hommes se font arrêter, des femmes prennent parfois leur place aux postes de responsabilité, comme dans le cas de Basanti Debi. La police riposte contre ces femmes, surtout dans les zones rurales, par des brutalités, des arrestations et des coups de feu.

\footnotetext{
28 Hin 1930. Également cité par Thapar-Björkert $2006: 43$.

29 Thapar-Björkert $2006: 55$.
} 
À partir des années 1930, les femmes commencent à s'engager politiquement de leur propre chef, sans y être poussées par les hommes de leur famille, et leur nombre dans le mouvement nationaliste ne fait qu'augmenter. Cependant, les femmes des classes moyennes et supérieures ne se mélangent pas aux femmes des classes laborieuses, des basses castes ou encore aux prostituées. Dans l'Uttar Pradesh, des membres du Comité de District du Congrès, estimant inconcevable que des femmes de classes moyennes côtoient des prostituées, interdisent donc à ces dernières de prendre part au mouvement. D'ailleurs, Gandhi leur refuse l'accès du Parti, à moins qu'elles ne changent de profession ${ }^{30}$. La plupart des hommes et des femmes des hautes castes ou classes, à l'instar de Gandhi, estiment que les prostituées ne sont pas assez morales pour lutter pour l'indépendance, et que leur participation ternirait la réputation du mouvement aux yeux des Britanniques. Quelques voix dissonantes cependant maintiennent qu'en se dévouant pour la cause nationaliste, les prostituées pourraient se racheter, devenir des femmes respectables, et se rapprocher du modèle de la maternité idéelle.

Les hommes du mouvement nationaliste insistent sur la respectabilité des femmes et sur le fait que leur engagement relève d'un devoir patriotique. En outre, la présence de femmes des classes moyennes et supérieures jouissant d'une réputation irréprochable renforce la cause. Dans l'Uttar Pradesh, des parentes de Jawahar Lal Nehru et d'Aruna Asaf Ali, militante active du Congrès, participent à la plupart des événements politiques. En évoquant dans ses discours politiques des produits d'usage courant, comme le sel ou le tissu, Gandhi contribue à attirer des femmes dans la lutte. Celles qui enfreignent la loi sur le sel (ce qu'on appelait la satyagraha du sel) sont sensibles à l'argument de Gandhi selon lequel on peut, en faisant preuve de force morale, changer le système. Un périodique de l'époque pose ainsi la question :

Quel rôle vont jouer les femmes? Vont-elles rester dans un coin de leur maison, dans les ténèbres de l'ignorance et dans la solitude, alors que la roue de la révolution est en train de tourner pour la dernière fois ? Ont-

30 Thapar-Björkert $2006: 98$. 
elles raison de croire que le champ de bataille est le domaine exclusif de l'homme et que le leur est le foyer ? ${ }^{31}$

Le boycott du sel, ingrédient essentiel de la vie quotidienne, parle à toutes et tous et représente ainsi un "contexte culturel partagé », contribuant à la naissance d'une identité nationale ${ }^{32}$. Le boycott débute le 12 mars 1930. À l'origine, Gandhi ne souhaite pas que des femmes y contribuent mais elles ne l'entendent pas ainsi. Khurshebdehn, petite-fille de Dadabhai Naoroji, l'un des principaux leaders du Congrès et Mridula Sarabhai, figure éminente du Congrès au Gujarat, formulent leurs exigences :

Aucune réunion ou commission ayant pour sujet le destin de l'Inde ne devrait avoir lieu sans la présence des femmes. De même, elles exigent qu'aucune manifestation, aucune marche, aucune incarcération dans un but similaire n'interdise la présence de femmes ${ }^{33}$.

Gandhi et ses homologues se rendent rapidement compte de la nécessité d'un soutien des femmes lorsqu'ils commencent à être jetés en prison. Gandhi et Abbas Tyabji ont choisi Sarojini Naidu pour attaquer les salines de Dharasana, à $250 \mathrm{~km}$ au nord de Bombay. Avant de prendre la tête de sa troupe, Naidu déclare :

À mon avis, le temps est venu pour les femmes de cesser de s'abriter derrière leur sexe. Elles doivent partager également avec leurs camarades les périls et les sacrifices nécessaires à la libération de leur pays ${ }^{34}$.

Les militantes nationalistes ont recours à divers moyens pour faire le blocus des magasins de tissu. Le plus souvent, elles vont et viennent devant les boutiques, encourageant les client-e-s à acheter du khadi. Elles poursuivent celles et ceux qui viennent d'acheter du textile importé pour les convaincre de rendre leur marchandise. Elles exigent même des marchands qu'ils promettent de ne plus vendre d'étoffe étrangère. En cas d'échec, elles se couchent devant l'entrée du magasin pour empêcher les clients d'entrer. Les Britanniques les appellent péjorativement «agitatrices professionnelles » ou « extrémistes». En 1930, Le Raj britannique renforce l'Ordonnance

31 Cité par Thapar-Björkert $2006: 102$.

32 Banjeree 2003 : 167-168.

33 Stree Dharma, vol. 13, 1930 : 247.

34 Times of India, 8 mai 1930. 
sur la Prévention de l'Intimidation, qualifiant de délits le boycott et «l'instigation illégale», piliers des actions devant les boutiques de tissu et d'alcool ${ }^{35}$. Les nationalistes sont en effet persuadés qu'il faut boycotter les magasins vendant des produits étrangers et de l'alcool, qui génèrent des revenus pour les Britanniques : et, de fait, boycott et piquets, auxquels ont largement participé les femmes, font chuter la vente de marchandises importées et d'alcool, et par contrecoup les revenus du gouvernement.

En dehors de ces actions, les femmes s'efforcent aussi de susciter une conscience nationaliste au travers des chants rituels de dévotion du matin (probhat pheris). Traditionnellement, aux petites heures du jour, des groupes de femmes et d'enfants entonnent des chants religieux entre le foyer et le temple. À l'époque de la mobilisation politique, ces chants prennent une tournure plus nationaliste que religieuse, et les chanteuses terminent ces rassemblements en réunions politiques plutôt que dans un temple. Pour mettre un terme aux probhat pheris, aux réunions et discours politiques, comme aux journaux en langue vernaculaire, les Britanniques limitent la liberté d'expression par le renforcement de la loi sur la presse et les livres, qui date de 1867. Sarojini Naidu affirme alors :

On peut toujours confisquer les presses, l'encre et les stylos mais on ne peut pas emprisonner un cœur qui aspire à la liberté. L'aspiration à la liberté est une vertu ${ }^{36}$.

Même après que Gandhi a mis un terme au mouvement de noncoopération, des Bengalies demeurent impliquées dans le mouvement nationaliste. Dans les années 1930 et 1940, celles qui doivent leur formation politique au mouvement anti-britannique poursuivent la lutte, parfois de façon violente. On ne saurait retracer l'histoire des femmes et du nationalisme en Inde sans évoquer le parcours de certaines d'entre elles.

35 Cité par Thapar-Björkert 2006 : 114 (dossier n¹51/1930. Police Department, Correspondence from Commissioner of Meerut Division to Secretary to the Government of the United Provinces).

36 Thapar-Björkert $2006: 119$. 


\section{Les femmes et le militantisme révolutionnaire : des années 1920 au mouvement pour la Liberté}

En même temps que le mouvement non-violent de Gandhi, se développe aussi un mouvement révolutionnaire, également tourné vers la résistance contre le colonisateur. Bien que cette idéologie ait existé ailleurs en Inde, c'est au Bengale qu'elle est la plus forte et la plus précoce (dès la fin du XIX siècle). Dans ce cas précis, l'idéologie rejoint un engouement pour la culture physique, qui s'est développé parmi les jeunes Bengalis, en réponse aux stéréotypes britanniques qui les décrivent comme efféminés ${ }^{37}$. On voit alors naître un différend au sein des classes moyennes et supérieures sur la question de l'indépendance. Une grande partie des Bengalis de la classe moyenne promeuvent la lutte armée contre les Britanniques, qui dès lors se mettent à décrire le Bengale comme « le foyer et le terrain d'action du terrorisme $»^{38}$. Mais une autre partie de ces catégories sociales est convaincue que l'indépendance ne peut s'obtenir que par des moyens non-violents. De nombreux Bengalis se trouvent des points communs avec les Irlandais opposés à l'administration britannique ${ }^{39}$.

L'implication des femmes dans les activités révolutionnaires est imputable à plusieurs facteurs. Dans l'Uttar Pradesh et au Bengale, les femmes sont très choquées par les condamnations à mort, le traitement infligé aux prisonniers et plus généralement par la violence des autorités britanniques. Déçues par le mouvement non-violent, certaines se tournent vers l'action révolutionnaire.

Jusqu'aux années 1930, les principales organisations révolutionnaires du Bengale admettent très peu de femmes mais, à partir de cette date, elles participent à parts égales à la lutte pour l'indépendance, dans tous les aspects de l'action révolutionnaire: assassinats ou raids contre des cibles coloniales ${ }^{40} \ldots$ Après le raid sur l'armurerie de Chittagong en 1930, une nouvelle loi permet d'emprisonner de nombreux jeunes Hindous. Les témoignages des

\footnotetext{
37 Russell 1980.

38 Hale 1974 [1937].

39 Au sujet de l'influence irlandaise sur les Bengalis, voir Basu 1995; O’Malley 2008 ; Thapar-Björkert \& Ryan 2002.

40 Chatterjee 2001 : 42 ; Bose 2003 : 155.
} 
participants au raid pendant leur procès montrent bien qu'ils tiraient une partie de leur inspiration de l'insurrection de Pâques 1916 en Irlande et des tactiques de guérilla de l'IRA ${ }^{41}$.

Les femmes qui mènent des activités révolutionnaires ne sont pas épargnées par la répression: le 14 décembre 1931, deux écolières adolescentes sont pendues pour avoir assassiné l'un des magistrats du district. Une autre femme, Pritilata Oaddedar, âgée de 21 ans, est la première martyre du mouvement nationaliste indien. Avec sept jeunes hommes, elle a attaqué le club européen de Chittagong (actuel Bengladesh), censément fréquenté par des administrateurs civils et policiers. Lorsqu'elle est capturée, elle se suicide, après avoir écrit : «La société indienne maintient par la force les femmes dans la sujétion. Mais en se vouant à la cause politique, elles ont démontré qu'elles n'étaient en rien inférieures $»^{42}$. Le 6 février 1932, Bina Das, alors âgée de 22 ans, tente d'assassiner le Gouverneur général du Bengale durant son allocution au Conseil de l'Université. Ayant raté sa cible, elle est arrêtée sur le champ et condamnée à neuf ans de travaux forcés. Libérée en 1939, elle entre au Congrès National Indien, avant d'être à nouveau emprisonnée durant trois ans pour participation au mouvement « Quit India ». De 1946 à 1951 enfin, elle est membre de la Législature du Bengale occidental ${ }^{43}$.

La Seconde Guerre mondiale amplifie la lutte puisqu'en tant que colonie britannique, l'Inde doit participer à l'effort de guerre. Après que le Japon a rejoint l'Axe par réaction contre les Britanniques, ceuxci se mettent à craindre une invasion japonaise de l'Inde. Pour la prévenir, les soldats britanniques pratiquent une politique de terre brûlée au Bengale, dont l'effet est de faire augmenter le prix des

41 Bose $2003: 135-139$.

42 Pritilata Oaddedar (1911-1932), née à Chittagong, prend la tête de l'école de filles du Bengale en 1932. À la même date, elle devient membre de l'Armée Républicaine de Chittagong et participe au raid contre le club européen. Cité par Chaudhuri 2001 : 37. La traduction est de l'auteure.

43 Bina Das est née en 1911. Son père était enseignant et sa mère travaillait dans le secteur social ; sa sœur aînée était également engagée dans la lutte armée contre les Britanniques. Lorsqu'elle obtient sa licence en 1931, cela fait trois ans qu'elle milite dans le mouvement révolutionnaire. Sa vie de révolutionnaire est consignée dans ses mémoires, intitulés Srinkholer Jhamkar (Le bruit des chaînes). 
denrées, de créer un marché noir - et de susciter un fort ressentiment anti-britannique, qui se diffuse progressivement dans toute l'Inde. Afin de satisfaire les revendications d'indépendance et de gagner en retour un véritable soutien durant la guerre, le gouvernement britannique propose de faire accéder le pays au statut de dominion une fois la guerre terminée. Mais le Parti du Congrès rejette cette offre et Gandhi entame la campagne "Quit India » à Bombay, le 8 août 1942. La riposte britannique ne tarde pas et la quasi totalité des chefs du Congrès sont arrêtés et incarcérés, hommes et femmes, au plan national ou local. Nombre d'entre eux passent le reste de la guerre en prison, ce qui ne fait qu'accroître le mécontentement populaire et augmenter le nombre d'actions violentes.

Ce mouvement fournit aux femmes une nouvelle occasion de rejoindre la lutte pour la liberté. Aruna Asaf Ali, membre du Congrès, opte pour la violence afin d'assurer le succès du mouvement nationaliste. Avec Sucheta Kripalani, une autre femme du Congrès, elles mobilisent et dirigent les masses, rédigent et distribuent tracts et bulletins sur les progrès du mouvement. Le gouvernement émet un avis de recherche à l'encontre de ces deux femmes, allant jusqu'à offrir une récompense. Après l'arrestation rapide de Kripalani, Aruna Saf Ali parvient à prendre la fuite et voyage dans tout le pays, visitant des zones frappées par la famine, aidant les villageois appauvris, et encourageant les familles de prisonniers politiques. Elle est aidée en retour par nombre de compatriotes, parmi lesquels des hommes d'affaires, des industriels, des fonctionnaires et des militaires ${ }^{44}$. La radio commence d'être utilisée pour étendre le mouvement. Avec des camarades, Usha Mehta, elle aussi membre du Congrès, acquiert un émetteur pour diffuser des nouvelles : une émission quotidienne est consacrée à démontrer que les Britanniques sont les ennemis de l'Inde et qu'il faut les chasser. Bientôt, l'émission est audible de Kolkata à Madras : au bout de trois mois, Usha Mehta et les autres sont arrêtés et condamnés à la prison à vie ${ }^{45}$.

Subhas Chandra Bose, Président élu du Congrès en 1938 et 1939 mais en désaccord avec Gandhi sur les modalités de la lutte pour

\footnotetext{
44 Agnew 1979: 73.

45 Agnew 1979: 74.
} 
l'indépendance, a dû quitter l'Inde et demeure en Asie du Sud-Est. En 1943, il met sur pied l'Armée Nationale Indienne (Azad Hind Fauj), qui comprend une division féminine, intitulée Rani du Jhansi, en référence à l'icône rebelle de la mutinerie de 1857. Il estime que ce régiment féminin constitue une «alternative légitime au courant nonviolent de Gandhi, représenté par Sita, le principe de soumission féminine ${ }^{46}$. Kesar Kaur et Lakshmi Swaminatham Sehgal sont deux figures majeures du régiment Rani du Jhansi. Après la mort mystérieuse de Bose et la défaite du Japon en 1945, l'Armée Nationale Indienne est défaite par les Britanniques et ses membres, notamment féminines, sont condamnés à la prison à vie. On voit à quel point l'idée très répandue selon laquelle le Congrès opta pour la lutte non-violente est infondée : il existait bel et bien une idéologie révolutionnaire, qui eut aussi un impact dans la lutte anticoloniale.

En effet, dès 1905, le mouvement nationaliste ou swadeshi avait incorporé des éléments de tactique violente, après que des militants, d'abord au Bengale puis au Punjab, aient fini par conclure que la stratégie non-violente se révélait inefficace. Cet élément de violence ne fit que croître à travers le pays, jusqu'à atteindre un premier sommet en 1920. Le soutien aux méthodes armées et l'abandon de la non-violence furent portés par de grandes figures de la société indienne. Ainsi, Rabindranath Tagore, le poète et prix Nobel, estime que la liberté ne peut résulter d'appels non-violents adressés au roi mais seulement de la lutte armée ${ }^{47}$. La cible de ce nouveau groupe de nationalistes est principalement constituée par les officiers britanniques, symboles de la tutelle coloniale; ainsi que par leurs soutiens dans la société indienne, souvent des commerçants hindous ou musulmans, que les révolutionnaires considèrent comme des traîtres. Nombre de femmes, gagnées à l'idée que la violence est le seul moyen de lutter pour l'indépendance, rejoignent des groupes revolutionnaires. La plupart d'entre elles, souvent instruites et issues de classes moyennes, n'ont pas recours aux explosifs ou aux armes, contrairement à leurs camarades masculins. Mais elles ont un rôle dans la promotion des activités révolutionnaires, dans le soutien aux

\footnotetext{
46 Hills \& Silverman $1993: 744$.

47 Chaudhuri 2001 : 20.
} 
militants pourchassés qu'elles cachent, dans la transmission de messages et d'informations, ou encore dans le transport d'armes à feu. En 1936, le Bureau de Renseignements du gouvernement britannique établit un répertoire intitulé «Le Terrorisme en Inde, 1917-1936». Peter Hees, auteur de l'ouvrage The Bomb in Bengal, a démontré que les cibles de ces révolutionnaires étant clairement sélectionnées, parmi les principaux responsables et non parmi les gens ordinaires, on ne pouvait parler de terrorisme mais bien de militantisme ${ }^{48}$. L'historienne Geraldine Forbes estime également que le qualificatif de « révolutionnaire » convient à ce mouvement, dans le sens où le terme bengali biplabi, signifie «lutte pour la liberté du pays »; elle rejette donc l'usage du terme « terroriste $»^{49}$.

Avec l'évolution du mouvement nationaliste, et le passage d'un mouvement non-violent à un mouvement mixte, composé d'éléments non-violents et de lutte armée, les femmes indiennes voient se modifier aussi leur opinion. Ayant trouvé des femmes prêtes à leur prêter main-forte, les militants révolutionnaires les intègrent sans équivoque comme des égales dans la lutte nationaliste. Tandis qu'elles contribuent à étendre et à renforcer la lutte armée, elles sont reconnues comme légitimes et même indispensables dans tout effort visant à la création d'un État-nation. Cette époque est décisive car de nombreuses femmes, s'étant fait reconnaittre comme égales dans la lutte, accèdent à des postes de responsabilité dans l'administration indienne juste après l'indépendance: à partir de 1947, elles sont nombreuses à être nommées dans des cabinets ministériels ou à des postes d'ambassadeurs. Lors des premières élections, en 1952, puis pendant les années suivantes, elles sont candidates au Parlement ou dans les Assemblées législatives d'États. Leur investissement dans le mouvement nationaliste a permis aux femmes de gagner beaucoup sur le plan politique ; et elles en profitèrent pour lancer le débat sur la question de l'égalité hommes/femmes, faisant en sorte que son principe soit reconnu dans la Constitution du pays.

Article traduit par Anne Hugon (Université Paris I, Cemaf)

48 Hees $2004:$ xvi.
49 Forbes $1980: 1$. 


\section{Sources primaires}

\section{Archives}

Report on the Native newspapers in Bengal, West Bengal State Archives, Calcutta.

\section{Ouvrages publiés}

BASU Sankari Prosad, 1985, Nivedita Lokamata (Nivedita, People's Mother), Kolkata, Ananda Publisher, p. 25-26.

BASU Sankari Prosad (ed.), 1982, Letters of Sister Nivedita, vol. I, Kolkata, Nababharat Publishers.

Besant Annie, 2008 [1893], An Autobiography, Rockville, Maryland, Arc Manor.

Chaudhurani Sarala Devi, 1975 [réédition], Jibaner Jharapata (Fallen Leaves of Life), Kolkata: Rupa, 1382 BS (Bengali century).

Cousins James H. \& Margaret E., 1950, We two, together, Madras, Ganesh.

DAS Krishnobhabini, 1885, Englande Bangamobila (A Bengali lady in England), written by a Bengali Lady, Calcutta, Sri Satya Prokash Sarbadhikari.

PravarajIKa Atmaparna (ed.), 1967, The Complete Works of Sister Nivedita: Birth Centenary Publications, Kolkata, Ramakrishna Sarada Mission, Sister Nivedita Girls' School.

\section{Journaux et périodiques}

PP. Hin. B.215, 1930. Sarojini Sandesha, in (proscribed book collection) British Library, London, U.K.

Vartman, Kashi.

Bamabodbini Patrika, Kolkata.

Stree Dharma, Madras.

The Times of India, Lucknow.

\section{Bibliographie}

Agnew Vijay, 1979, Elite Women in Indian Politics, New Delhi, Vikash Publishing House.

AmIN Sonia Nishat, 1995, "The Early Muslim Bhadramahila: The Growth of Learning and Creativity, 1876-1939", in Bharati RAY (ed.), From the Seems of History. Essays on Indian Women, Oxford, Oxford University Press, p. 107-148.

ANGol Padma, 2006, The Emergence of Feminism in Western India, Londres, Ashgate.

ANTHias Floya \& Nira Yuval-Davis, 1989, Women-Nation-State, Londres, Macmillan.

BANERJEE Sikata, 2003, « Gender and Nationalism: The Masculinisation of Hinduism and Female Political Participation in India ", Women's Studies International Forum, 935/1, p. 167-179.

BASU Sharmila, 1995, «Bidrohi Banddhon: Ireland O Bharat », Desh, 62, p. 37-49. 
BASU Aparna \& Bharati RAY, 1990, Women's Struggle: A History of All India Women's Conference, 1927-1997, Delhi, Manohar Publications.

Blom Ida, 2000, "Gender and Nation in International Comparison » in Ida BLOM, Karen Hagemann \& Catherine Hall (eds), Gendered Nations: Nationalisms and Gender Order in the Long Nineteenth-Century, Berg, New York.

BORTHwiCK Meredith, 1984, The Changing Role of Women in Bengal, 1849-1905, Princeton, Princeton University Press.

Bose Purnima, 2003, Organizing Empire: Individualism, Collective Agency and India, Durham, Duke University Press.

BurTON Antoinette, 1994, Burdens of History: British Feminists, Indian Women and Imperial Culture, 1865-1914, Chapel Hill, University of North Carolina Press.

-, 1998, At the Heart of the Empire: Indians and the Colonial Encounter in Late-Victorian Britain, Berkeley, University of California Press.

—, 2003, Dwelling in the Archive: Women Writing House, Home and History in Late Colonial India, New York, Oxford University Press.

Chapman-Lebra Joyce, 1986, The Rani of Jhansi: A Study in Female Heroism in India, Honolulu, University of Hawaii Press.

Chatterjee Manini, 2001, «1930: Turning Point in the Participation of Women in the Freedom Struggle », Social Scientist, vol. 29 : 7-8, p. 39-47.

Chaudhuri Chinmoy, 2001, Swadhinata Andolone Shabingsa Nari Violent Women in Freedom Movement), Calcutta, Dey's Publishing.

Chaudhuri Nupur \& Margaret STROBel, 1992, Western Women and Imperialism: Complicity and Resistance, Bloomington, Indiana University Press.

Chaudhuri Nupur, 2000, "Issues of Race, Gender and Nation in English Women's Domestic Magazine and The Queen, 1850-1900 ", in David FINKELSTEIN \& Douglas M. PeErs (eds), Negotiating India in the Nineteenth Century Media, London, Macmillan, p. 51-62.

-, 2010, "Finding an Archive in Krishnobhabini Das's Englande Bangamobila", in Nupur Chaudhuri, Sherry J. Katz \& Mary E. Perry (eds), Contesting Archives: finding Women in the Sources, Urbana, University of Illinois Press, p. 135-155.

FORBES Geraldine, « Goddesses or Rebels? The Women Revolutionaries of Bengal », The Oracle, 2/2, p. 1-15.

—, 1998, Women in Modern India, Cambridge, Cambridge University Press.

GREWAL Inderpal, 1996, Home and harem: nation, gender, empire, and the cultures of travel, Durham, Duke University Press.

Hale H.W., 1974 [1937], Terrorism in India, 1917-1936, Allahabad, Chugh Publications.

Hees Peter, 2004, The Bomb in Bengal, New Delhi, Oxford University Press.

Hills Carol \& Daniel Silverman, 1993, "Nationalism and Feminism in Late Colonial India: The Rani of Jhansi Regiment, 1943-1945», Modern Asian Studies, 27/4, p. 741-760. 
Kosambi Meera, 2000, Pandita Ramabai, Through Her Own Words: Selected Work, India, Oxford University Press.

Levine Philippa (ed.), 2004, Gender and Empire, New York, Oxford University Press.

O'Malley Kate, 2008, Ireland, India and Empire: Indo-Irish Radical Connections, 1919. 1964, Manchester, Manchester University Press.

RAMUSACK Barbara, 1981, «Catalysts or Helpers? British Feminists, Indian Women's Rights and Indian Independence », in Gail Minault (ed.), The Extended Family: Women and Political Participation in India and Pakistan, Columbia, Mo., South Asia Books.

—, 1992, "Cultural Missionaries, Maternal Imperialists, Feminist Allies », in Nupur Chaudhuri \& Margaret STROBEL (eds), Western Women and Imperialism, Bloomington, Indiana University Press, p. 119- 136.

RAY Bharati, 2002, Early Feminists of Colonial India: Sarala Devi Chaudhurani and Rokaya Sakhawat Hossain, New Delhi, Oxford University Press.

RAY Bharati (ed.), 1995, From the Seams of History: Essays on Indian Women, New Delhi, Oxford University Press.

Russell John, 1980, "The Self-Image of Effeteness: Physical Education and Nationalism in Nineteenth-Century Bengal », Past and Present, 86/2, p. 121-148.

SARKAR Tanika, 1993, «A Book of Her Own, A Life of Her Own: Autobiography of a Nineteenth-Century Woman ", History Workshop Journal, 36, p. 35-65.

—, 1999, The Words to Win: The Making of Amar Jiban, A Modern Autobiography, New Delhi, Kali for Women.

- 2001, Hindu Wife, Hindu Nation: Community, Religion, and Cultural Nationalism, Bloomington, Indiana University Press.

Silvestri Michael, 2000, "The Sinn Féin of India": Irish Nationalism and the Policing of Revolutionary terrorism in Bengal », Journal of British Studies, 39/4, october, p. 460-461.

SINDHE Rosalind Tarabai, 1994, A Comparison between Women and Men, transl. Rosalind O. Hanlon, New Delhi, Oxford University Press.

SINHA Mrinalini, 1996, "Gender in the Critiques of Colonialism and Nationalism: Locating the "Indian Woman" " in Joan W. SCOTT (ed.), Feminism and History, New York, Oxford University Press.

SreenIVAs Mytheli, 2008, Wives, Widows, and Concubines: The Conjugal Family Ideal in Colonial India, Bloomington, Indiana University Press.

THAPAR-BJÖRKERT Suruchi, 2006, Women in the Indian National Movement: Unseen Faces and Unheard Voices, 1930-1942, New Delhi, Sage.

THAPAR-BJÖRKERT Suruchi \& Louise RYAN, 2002, « Mother India/Mother Ireland: Comparative gendered dialogues of colonialism and nationalism in the early $20^{\text {th }}$ century ", Women's Studies International Forum, 25/3, p. 301-313.

WoollacotT Angela, 2006, Gender and Empire, New York, Palgrave. 